\title{
Quark asymmetries in the proton from a model for parton densities
}

\author{
J. Alwall* and G. Ingelman ${ }^{\dagger}$ \\ High Energy Physics, Uppsala University, Box 535, S-75121 Uppsala, Sweden \\ (Received 11 March 2005; published 18 May 2005)
}

\begin{abstract}
Based on quantum fluctuations in momentum and of the proton into meson-baryon pairs, we develop a physical model for the nonperturbative $x$ shape of parton density functions in the proton. The model describes the proton structure function and gives a natural explanation of observed quark asymmetries, such as the difference between the anti-up and anti-down sea quark distributions and between the up and down valence distributions. An asymmetry in the momentum distribution of strange and antistrange quarks in the nucleon is found to reduce the $\mathrm{NuTeV}$ anomaly to a level which does not give a significant indication of physics beyond the standard model.
\end{abstract}

DOI: 10.1103/PhysRevD.71.094015

PACS numbers: $12.39 . \mathrm{Ki}, 11.30 . \mathrm{Hv}, 13.15 .+\mathrm{g}, 13.60 . \mathrm{Hb}$

\section{INTRODUCTION}

The parton distributions in hadrons play a very important role in particle physics. Cross sections for hard processes involving incoming hadrons can, based on the factorization theorems of QCD [1], be calculated as a convolution of the parton distributions with parton level cross sections calculated using perturbation theory. The parton distributions are universal in the sense that each hadron has a unique parton structure which can be used to calculate all hard processes involving that hadron. The normal interpretation of a parton distribution $f_{i}\left(x, Q^{2}\right)$ is as the probability to find a parton $i$ (quark of some flavor or gluon) with a fraction $x$ of the hadron momentum when probed by the momentum transfer $Q^{2}$. The $Q^{2}$ dependence is very successfully described in perturbative QCD (PQCD) by the $\log Q^{2}$ evolution equations [2], which means that, given the input distributions in $x$ at a scale $Q_{0}^{2}$ large enough for PQCD to be applicable, one can calculate the distributions at any higher $Q^{2}$.

However, this starting $x$ shape, which depends on nonperturbative QCD dynamics of the bound state hadron, has not yet been successfully derived from first principles. Instead, they are obtained by fitting parametrizations to data, in particular, structure function measurements in deep inelastic lepton-nucleon scattering, e.g. the CTEQ [3] and MRST [4] parametrizations.

Here we present further developments of our phenomenological model [5] used to derive the parton distributions from simple assumptions regarding the nonperturbative properties of the hadron. The basic idea is to define the valence parton momentum distributions in the hadron rest frame, where we assume that they are described by spherically symmetric Gaussians. The typical width of these distributions is a few hundred MeV from the Heisenberg uncertainty relation applied to the hadron size. Sea partons are described through quantum fluctuations of the nucleon into baryon-meson pairs, having the same quantum num-

\footnotetext{
*Electronic address: johan.alwall@tsl.uu.se

†Electronic address: gunnar.ingelman@tsl.uu.se
}

bers as the original nucleon, and the sea parton is identified with a valence parton in the fluctuation meson. Through this mechanism, asymmetries in the nonperturbative sea parton distributions naturally arise, such as the difference between the $\bar{u}$ and $\bar{d}$ distributions and an asymmetry in the momentum distribution of $s$ compared to $\bar{s}$.

The explanation of asymmetries in the nucleon sea by fluctuations of the nucleon into baryon-meson pairs also occurs in "meson cloud" models as reviewed in Ref. [6]. Although the meson cloud models have an elaborate theoretical formalism (see e.g. [7]), one is forced to introduce phenomenological parts in order to obtain numerical results that can describe data. This concerns the choice of form factor in the nucleon to baryon-meson $(N \rightarrow B M)$ splitting function and the parton distributions in both mesons and baryons. Our model invokes similar hadronic fluctuations as a basic quantum phenomenon but differs from meson cloud models in important aspects. We use Gaussian momentum distributions both for hadrons in the fluctuations and for the partons in the hadrons, and the hadronic fluctuations are considered only at the low starting scale $Q_{0}^{2}$, where the starting parton densities are defined and then evolved using standard perturbative QCD evolution. Thus, we have physically motivated parton momentum distributions in the nucleon as well as in the fluctuation hadrons, while meson cloud models usually use parametrizations. Our model results in a complete set of parton density functions, which we compare with different experimental data sets giving insights into different aspects of the nonperturbative nucleon structure.

In Sec. II we develop the details of the model and in Sec. III we compare to the relevant experimental data as well as to conventional parametrizations of parton densities. Finally, Sec. IV gives a concluding discussion.

\section{THE MODEL}

\section{A. Valence distributions}

This work is an extension of the previously presented physical model [5], giving the momentum distributions of partons in the nucleon, as illustrated in Fig. 1. The model 


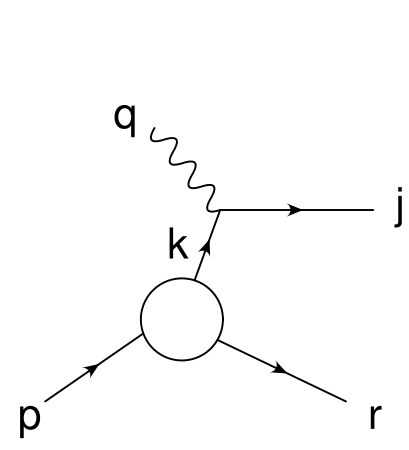

(a)

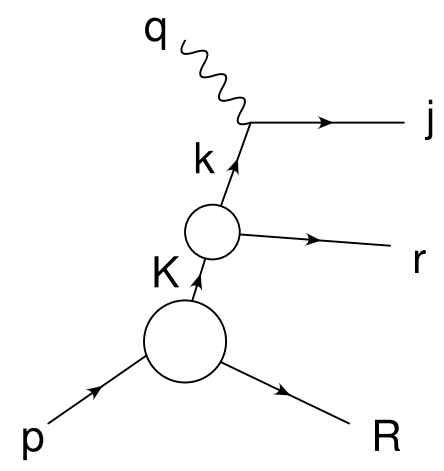

(b)
FIG. 1. Probing a valence parton in the proton and a sea parton in a hadronic fluctuation (letters are four-momenta).

gives the $x$ shape of the parton distributions of a hadron of mass $M$ at a momentum transfer scale $Q_{0} \approx 1 \mathrm{GeV}$, i.e. $x q\left(x, Q_{0}^{2}\right)$ and $x g\left(x, Q_{0}^{2}\right)$, which provide an effective description of the nonperturbative dynamics of the bound state nucleon. Our approach is not intended to provide the full wave function for the hadron but gives the fourmomentum $k$ of a single probed parton, whereas all other partons are treated collectively as a single remnant with four-momentum $r$ (see Fig. 1). In the nucleon rest frame, there is no preferred direction, and, hence, the parton momentum distribution is spherically symmetric. The shape of the momentum distribution for a parton of type $i$ and mass $m_{i}$ is then taken as a Gaussian

$f_{i}(k)=N\left(\sigma_{i}, m_{i}\right) \exp \left(-\frac{\left(k_{0}-m_{i}\right)^{2}+k_{x}^{2}+k_{y}^{2}+k_{z}^{2}}{2 \sigma_{i}^{2}}\right)$,

where $N$ is the normalization. The Gaussian form is not only a reasonable first guess but can be motivated as the collective result of the many small momentum transfers affecting the parton through the nonperturbative bound state interactions which cannot be calculated properly. The width $\sigma_{i}$ of the distribution should be of order hundred $\mathrm{MeV}$ from the Heisenberg uncertainty relation applied to the nucleon size, i.e. $\sigma_{i}=1 / d_{N}$. This Fermi motion inside the nucleon provides the "primordial transverse momentum," which has been extracted from deep inelastic scattering data and found to be well described by a Gaussian distribution of a few hundred $\mathrm{MeV}$ width [8] giving phenomenological support for this description.

The energy component does not have the same simple connection to the Heisenberg uncertainty relation. To keep the model simple and reduce the number of parameters, we assume a Gaussian distribution around the parton mass with the same width as the momentum fluctuations, such that partons can be off-shell at a soft scale of the binding interactions. This means a parton fluctuation lifetime corresponding to the nucleon radius.

The momentum fraction $x$ of the parton is then defined as the light-cone fraction $x=k_{+} / p_{+}$. Here, four-momenta are expressed as $p=\left(p_{+}, p_{-}, \vec{p}_{\perp}\right)$ where the "plus" and "minus" components are $p_{ \pm}=E \pm p_{z}$ and the $z$ axis defined by the probe. The fraction $x$ is then invariant under boosts along the $z$ axis and equivalent to the conventional momentum fraction $x=k_{z} / p_{z}$ in a frame where $p_{z}$ is large ("infinite momentum" frame).

In order to obtain a kinematically allowed final state, one must impose the following constraints. The scattered parton must be on-shell or have a timelike virtuality (causing final state QCD radiation) and an invariant mass less than the invariant mass of the hadronic system. Furthermore, the hadron remnant $r$ is obtained from energy-momentum conservation and must be timelike in order to project it on a final hadronic state through hadronization. Referring to Fig. 1(a) for the definitions of the momenta, one thus obtains:

$$
\begin{aligned}
m_{i}^{2} & \leq j^{2}=(k+q)^{2}<W^{2}=(p+q)^{2}, \\
r^{2} & =(p-k)^{2}>0 .
\end{aligned}
$$

An important consequence of these constraints is to ensure that $0<x<1$ and $f(x) \rightarrow 0$ as $x \rightarrow 1$.

The parton distributions are obtained by integrating Eq. (1) with these conditions. Using a Monte Carlo method, this can be achieved numerically without approximations. For the simple case of the valence distributions, it is possible to derive an analytical expression as well; see Sec. II B below. The normalization of the valence distributions is provided by the sum rules

$$
\int_{0}^{1} d x u_{v}(x)=2 \text { and } \quad \int_{0}^{1} d x d_{v}(x)=1
$$

to get the correct quantum numbers of the proton (and similarly for other hadrons). The normalization of the gluon distribution is chosen so as to saturate the momentum sum rule

$$
\sum_{i} \int_{0}^{1} d x x f_{i}(x)=1
$$

where the sum is over the valence quarks and gluons.

\section{B. Analytical expressions}

By integrating $f(k)$ in Eq. (1) over $k_{\perp}^{2}$ and $k_{-}$of the parton between the limits given by (2), we arrive at the following analytical expression for the bare hadron valence distributions:

$$
\begin{aligned}
f_{i}(x)= & N\left(\tilde{\sigma}_{i}, x_{i}\right)\left\{\left[1+\operatorname{erf}\left(\frac{1-x_{i}}{2 \tilde{\sigma}_{i}}\right)\right] \exp \left[-\frac{\left(x-x_{i}\right)^{2}}{4 \tilde{\sigma}_{i}^{2}}\right]\right. \\
& \left.-\left[1+\operatorname{erf}\left(\frac{x-x_{i}}{2 \tilde{\sigma}_{i}}\right)\right] \exp \left[-\frac{\left(1-x_{i}\right)^{2}}{4 \tilde{\sigma}_{i}^{2}}\right]\right\},
\end{aligned}
$$

where $\tilde{\sigma}_{i} \equiv \sigma_{i} / M, x_{i} \equiv m_{i} / M$, and $\operatorname{erf}(x)=(2 / \sqrt{\pi}) \times$ $\int_{0}^{x} d t e^{-t^{2}}$ (the error function). The normalization 
$N\left(\tilde{\sigma}_{i}, x_{i}\right)$ is given by (3). This analytical expression can be simplified when $\sigma_{i}$ and $m_{i}$ are sufficiently small (e.g. $\left.\sigma_{i}, m_{i} \lesssim M / 4\right)$ to

$$
\begin{aligned}
f_{i}(x)= & N\left(\tilde{\sigma}_{i}, x_{i}\right)\left[1-\exp \left(\frac{\left(1-x_{i}\right)(1-x)}{2 \tilde{\sigma}_{i}^{2}}\right)\right] \\
& \times \exp \left(-\frac{\left(x-x_{i}\right)^{2}}{4 \tilde{\sigma}_{i}^{2}}\right) .
\end{aligned}
$$

The "bare" proton valence $u$-quark momentum distribution $x u_{0 v}(x)$ given by Eq. (5) is shown by the solid curve in Fig. 2. The approximation in Eq. (6) gives an indistinguishable result and is therefore not shown explicitly. Figure 2 also demonstrates a very close agreement with the Monte Carlo simulation result of the model (histogram).

This behavior of the valence distributions is modified by the contributions from the baryons in the hadronic fluctuations described below. At $x \geqslant 0.7$ the shape of the valence distributions are still given exclusively by the original hadron, i.e. by Eq. (5), but at lower $x$ the valence distributions are significantly modified. The shift of the distribution to lower $x$ is clearly seen in Fig. 2 as the difference between the bare $x u_{0 v}(x)$ distribution and the "full" $x u_{v}(x)$ valence $u$-quark momentum distributions. A similar end result can also be obtained without including this baryon

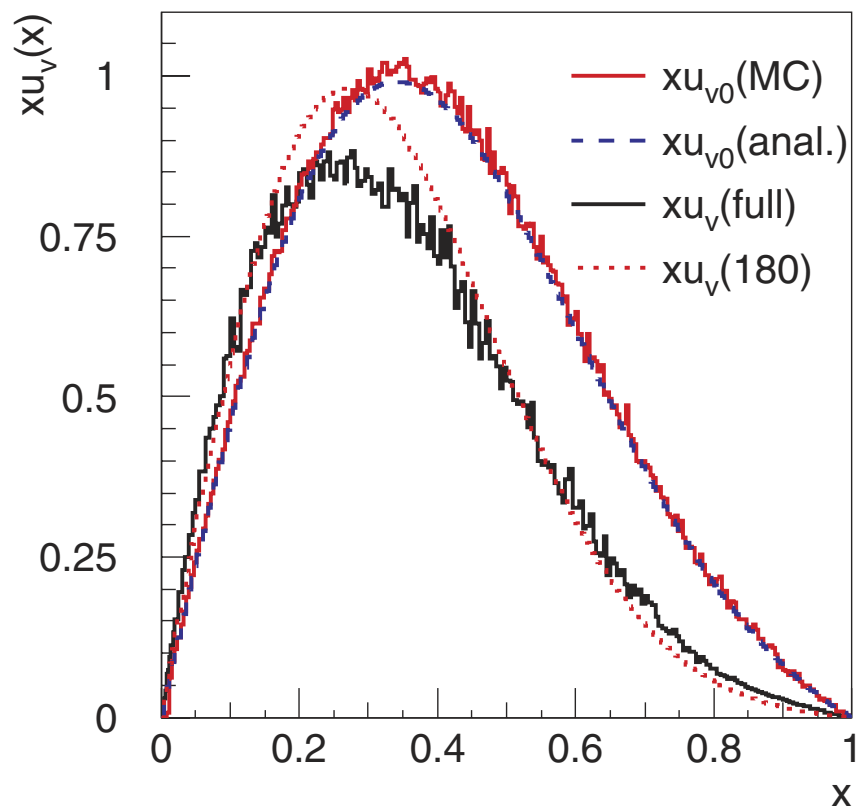

FIG. 2 (color online). Valence $u$-quark distribution of the proton obtained from the model: the bare proton distribution $x u_{v 0}(x)$ from Monte Carlo simulation compared to the analytical form Eq. (5), as well as the full $u_{v}$ distribution including simulation of partons in baryons in hadronic fluctuations $|p\rangle \rightarrow$ $|B M\rangle$ as described in Sec. IIC below. The dotted line is the valence distribution of Ref. [5], having a lower width $\sigma_{u}=$ $180 \mathrm{MeV}$ but no effect of baryons in fluctuations. [All curves show the momentum density, whereas the normalization is in the number density according to Eq. (3).] fluctuation effect, as in [5], by a lower Gaussian width when fitting the model to $F_{2}$ data (discussed below in Sec. III A).

One can note that the asymptotic behavior for very large $x \gtrsim 0.9$ is $(1-x)^{1}$ according to Eq. (5). For $0.3 \leqq x \leqq$ 0.9 , however, the $u$ quark distribution is well described by a form $u(x)=8(1-x)^{2.8}$, in good agreement with the $(1-x)^{3}$ dependence predicted by spectator counting rules [9].

\section{Hadronic fluctuations and sea distributions}

Sea partons arise from the nonperturbative dynamics of the bound state hadron, for which it should be appropriate to use a hadronic quantum mechanical basis. Therefore, we consider hadronic fluctuations of the proton

$$
\begin{aligned}
|p\rangle= & \alpha_{0}\left|p_{0}\right\rangle+\alpha_{p \pi}\left|p \pi^{0}\right\rangle+\alpha_{n \pi}\left|n \pi^{+}\right\rangle+\ldots \\
& +\alpha_{\Lambda K}\left|\Lambda K^{+}\right\rangle+\ldots,
\end{aligned}
$$

where the different states are assumed to be orthonormal and the normalization constants are real and fulfill $\alpha_{0}^{2}+$ $\sum_{B M} \alpha_{B M}^{2}=1$.

Probing a parton $i$ in a hadron $H$ of such a fluctuation [Fig. 1(b)] gives a sea parton with light-cone fraction $x=$ $x_{H} x_{p}$ of the target proton; i.e. the sea distributions are obtained from a convolution of the momentum $K$ of the hadron and the momentum $k$ of the parton in that hadron. We assume that the momentum $\vec{K}$ of the probed hadron is given by a similar Gaussian as in Eq. (1), with a separate width parameter $\sigma_{H}$ (for simplicity and definiteness, we make this parameter common for all fluctuations). The momentum $\vec{K}^{\prime}$ of the other hadron in the fluctuation is then fixed by momentum conservation in the rest frame of the original nucleon. We let both hadrons be on-shell, which fixes their energies. This implies that energy is not conserved at this intermediate stage but is, of course, restored for the observable final state. With the hadron four-vectors specified, one obtains the light-cone fraction $x_{H}=K_{+} /\left(K+K^{\prime}\right)_{+}$. A few possible modifications of the details of the model are discussed in the next section, and the possible use of an effective meson mass instead of the physical pion mass is discussed in Sec. III C.

The above model for valence distributions is then applied to the fluctuation hadron $H$ to get the parton momentum and light-cone fraction $x_{p}=k_{+} / K_{+}$in $H$. The flavor sum rules in Eq. (3) must, of course, be modified to apply for $H$. The kinematical constraints of Eq. (2) are in this case modified to

$$
\begin{aligned}
m_{i}^{2} & \leq j^{2}<W_{H}^{2}=(K+q)^{2}, \\
r^{2} & >0 \text { and }(r+R)^{2}=(p-k)^{2}>0,
\end{aligned}
$$

such that the scattered parton and the remnants [cf. Fig. 1(b)] are on-shell or have positive virtualities within 
physically allowed limits and, hence, can give a proper final hadronic state.

A Monte Carlo method is used to simulate this two-step process by choosing $K$ and $k$, impose the constraints, and obtain the momentum fraction $x$. By iterating the procedure, the additional quark and gluon distributions due to the fluctuations are generated. Note that the flavor number density $\int_{0}^{1} d x f_{i}(x)$ is not affected by the convolution with the fluctuation momentum. This means that the normalization criteria (3) (modified for the hadron in question) automatically ensure flavor conservation, since the meson and the baryon in the fluctuation are multiplied by the same normalization constant $\alpha_{B M}^{2}$.

Since the $x$ distribution of the fluctuation hadrons is given by $x_{H}=K_{+} /\left(K+K^{\prime}\right)_{+} \approx M_{H} /\left(M_{B}+M_{M}\right)$, the low- $x$ sea is mainly given by scattering off the meson in the baryon-meson fluctuation. The scattering off of the baryon in the baryon-meson fluctuation gives a contribution resembling the valence distribution but at lower $x$ than the bare hadron distribution. This means that the analytical valence distribution of the bare hadron derived above is modified for $x \lessgtr 0.7$ with the inclusion of hadronic fluctuations, as shown in Fig. 2.

The normalization of the sea distributions is given by the amplitude coefficients $\alpha_{B M}^{2}$. These partly depend on Clebsch-Gordan coefficients and should also include a suppression for larger masses. However, the full dependence is due to nonperturbative dynamics that cannot be calculated from first principles in QCD, including the possibility of mixing of different mesons/baryons with the same quark content. We therefore take the normalizations $\alpha_{B M}^{2}$ as free parameters.

\section{Alternatives in the definitions of fluctuation momenta}

In the details of the model described above, we have made some choices which could be done differently:

(1) In the definition of the fluctuation momentum, the fluctuation hadrons were made on-shell. Another possibility would be to let the energy of the probed hadron fluctuate around the hadron mass as in Eq. (1), allowing the fluctuation hadrons to be offshell on a soft scale. The differences in the resulting sea distributions are, however, small and can be mimicked by changing parameter values.

(2) We defined the upper bound on the scattered parton virtuality as $j^{2}<W_{H}^{2}=(K+q)^{2}$. This choice keeps the parton distributions in the fluctuation hadrons close to unaltered with respect to the distributions in the corresponding free hadrons. However, this choice allows the struck parton to have essentially all the proton momentum, which might seem unnatural. An alternative approach would be to put the upper bound on $j^{2}$ to $\left(x_{H} P+q\right)^{2} \simeq x_{H} W^{2}$, such that it is restricted by the longitudinal momentum fraction taken by the fluctuation hadron. This results in a softer meson momentum spectrum, because the distributions in the fluctuation meson are distorted. For completeness, we will consider both alternatives in our predictions for the $s-\bar{s}$ asymmetry (Sec. III D).

(3) The limits on the remnant momenta were put to $r^{2}>$ 0 (to ensure that $f(x) \rightarrow 0$ as $x \rightarrow 1)$ and $(r+R)^{2}=$ $(p-k)^{2}>0$. This latter choice is motivated by the fact that the fluctuation hadrons are close in space, such that an exchange of momentum on a soft scale should be possible, and, therefore, the condition is on the whole remnant system. Another possibility would be to force $r$ and $R$ separately to be timelike. This is obviously not possible if we scatter on a baryon with mass larger than the original hadron mass and make the fluctuation hadrons on-shell, since $R^{2}>0$ can then never be satisfied. In the off-shell case discussed under point 1 , this is possible and gives slightly harder meson spectra for low meson masses, whereas for meson masses $\gtrsim$ $300 \mathrm{MeV}$ the difference is negligible.

\section{GVDM contributions at low $Q^{\mathbf{2}}$}

In the same spirit as the hadronic fluctuations of the proton described above in Eq. (7), one should also note that a photon may appear as a vector meson, such that the quantum state should be expressed as

$$
|\gamma\rangle=C_{0}\left|\gamma_{0}\right\rangle+\sum_{V} \frac{e}{f_{V}}|V\rangle+\int_{m_{0}} d m(\cdots) .
$$

In the original vector meson dominance model (VDM) only fluctuations to vector mesons were considered [10], but in generalized models (GVDM), a contribution from a continuum of higher-mass states is also included [11], represented in Eq. (9) by an integral over masses.

The GVDM interpretation of the photon-proton interaction is illustrated in Fig. 3. The corresponding cross section is given by a convolution of the photon-to-meson fluctuation probability with the meson propagator and the mesonproton cross section [11], and the continuum contribution is included using a phenomenologically chosen spectral weight function. As shown in Ref. [12], the resulting

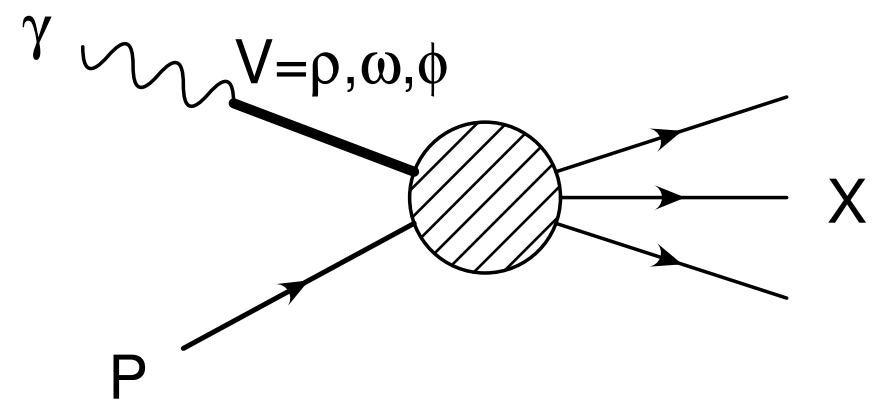

FIG. 3. Schematic view of the photon fluctuating to a vector meson before interacting with the proton. 
expression for the proton structure function,

$$
\begin{aligned}
F_{2}\left(x, Q^{2}\right)= & \frac{(1-x) Q^{2}}{4 \pi^{2} \alpha}\left\{\sum_{V=\rho, \omega, \phi} r_{V}\left(\frac{m_{V}^{2}}{Q^{2}+m_{V}^{2}}\right)^{2}\right. \\
& \times\left(1+\xi_{V} \frac{Q^{2}}{m_{V}^{2}}\right)+r_{C}\left[\left(1-\xi_{C}\right) \frac{m_{0}^{2}}{Q^{2}+m_{0}^{2}}\right. \\
& \left.\left.+\xi_{C} \frac{m_{0}^{2}}{Q^{2}} \ln \left(1+\frac{Q^{2}}{m_{0}^{2}}\right)\right]\right]_{A_{\gamma}} \frac{Q^{2 \epsilon}}{x^{\epsilon}},
\end{aligned}
$$

describes data very well for $Q^{2} \lesssim 0.7 \mathrm{GeV}^{2}$.

At higher $Q^{2}$ this GVDM contribution should be phased out in order to conform to the conventional description in terms of parton density functions. One way of doing this [12] is by introducing a phenomenological form factor $\left(Q_{C}^{2} / Q^{2}\right)^{a}$ on the GVDM contribution for $Q^{2}>Q_{C}^{2}$, which gives a good description of HERA $F_{2}$ data at these intermediate $Q^{2} \lesssim 4 \mathrm{GeV}^{2}$. The GVDM component was found to be negligible for $Q^{2} \geq 4 \mathrm{GeV}^{2}$.

The details of the phasing out of the GVDM component are not known and would require additional assumptions and parameters in the model. Fortunately, GVDM (giving symmetric quark-antiquark contributions) can be neglected in this study of the asymmetries in the proton, since the relevant data used in the following is at $Q^{2} \gtrsim 4 \mathrm{GeV}^{2}$, where the GVDM component was found to be negligibly small.

\section{RESULTS AND COMPARISONS TO EXPERIMENT}

With this simple model, based only on Gaussian momentum fluctuations and hadronic quantum fluctuations

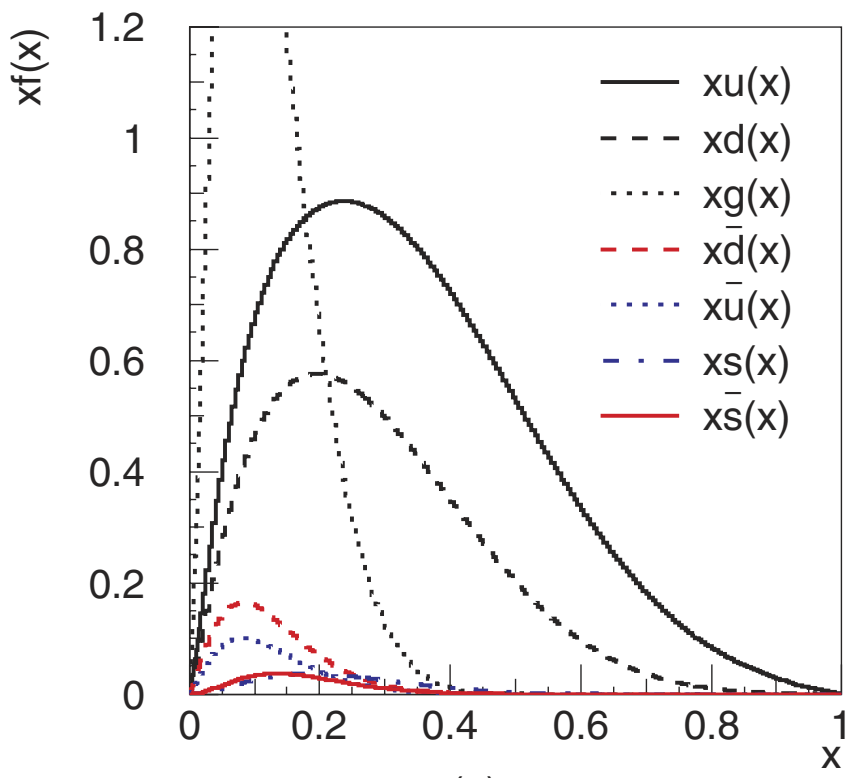

(a) together with kinematical constraints, we are able to describe several different aspects of the parton distribution functions of the nucleon. Perhaps phenomenologically most interesting, we describe the asymmetries between $\bar{u}$ and $\bar{d}$ in the proton quark sea. Also, we get an asymmetry in the nucleon strange sea, such that the $s$ quark has a harder momentum distribution than the $\bar{s}$ antiquark, which is of interest [13] in relation to the NuTeV anomaly [14].

Since we have most data on the proton, the parton widths are fitted for this special case of a hadron. For other hadrons, we assume for simplicity that the widths of the gluon distribution are the same as those for the proton. For valence quarks, we assume that the $d$ quark width is the typical width for a quark with quark number $\int q(x) d x=1$ in a hadron, while the $u$ quark width is typical for a quark with number $\int q(x) d x=2$. This means that in all mesons and also e.g. the $\Lambda$ baryon, all valence quark widths are given by the proton $d$ quark width. This can be motivated by the hadrons having essentially the same spacial extent, making the only relevant difference the possible effects of the Pauli exclusion principle. Such "Pauli blocking" (see [6] and references therein) would reduce the effective space available for the $u$ quarks in the proton, resulting in a larger momentum fluctuation width. Therefore, for the neutron we simply make an isospin transformation $d \leftrightarrow u$ of the proton distribution parameters.

The model provides valence and sea parton $x$ distributions as shown in Fig. 4. These apply at the low starting scale $Q_{0}^{2}$, while the parton distributions and the proton structure function $F_{2}\left(x, Q^{2}\right)$ at higher $Q^{2}$ are obtained by applying standard QCD evolution as implemented in the QCDNUM16 code [15]. For the results below, we have used

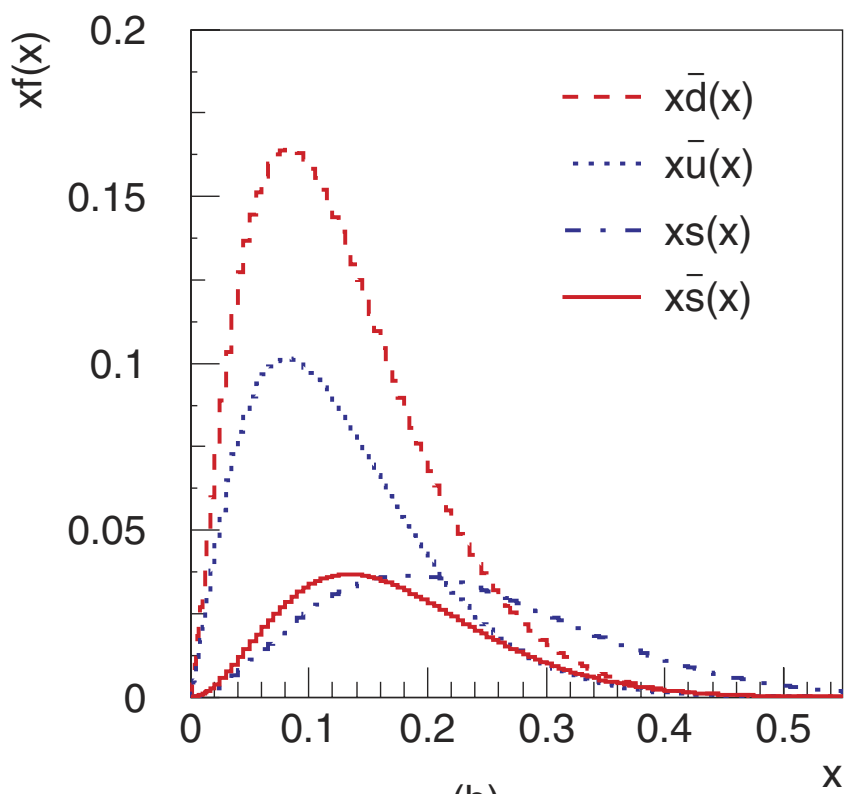

(b)

FIG. 4 (color online). Parton distributions from our model at the starting scale $Q_{0}^{2}$ : (a) all parton distributions, (b) the sea parton distributions (note the different scales). 
the conventional $\overline{M S}$ scheme and the evolution equations in next-to-leading order to have the highest available theoretical accuracy. Using the option of only leading order equations, the values of all model parameters are unaffected except $Q_{0}$ and $\sigma_{g}$ as discussed in Sec. III E. With leading order evolution, however, the quality of the fit to data becomes worse.

Since different model parameters are sensitive to different data, we have used data from several different experiments:

(i) fixed-target $F_{2}$ data $[16,17]$ to fix large- $x$ (valence) distributions;

(ii) HERA $F_{2}$ data [18] for the gluon distribution width and the starting scale $Q_{0}^{2}$;

(iii) $W^{ \pm}$charge asymmetry data [19] as a cross-check on the ratio of Gaussian widths for the $u$ and $d$ valence quark distributions;

(iv) $\bar{d} / \bar{u}$-asymmetry data [20] for the normalizations of the $\left|p \pi^{0}\right\rangle$ and $\left|n \pi^{+}\right\rangle$fluctuations; (v) strange sea data [21] to fix the normalization of the fluctuations including strange quarks.

It should be noted, however, that most of the model parameters influence several observables at least to some degree, making the task of a total fit quite involved.

It is interesting to note that this simple model can describe such a wealth of different data with just one or two parameters per data set, as will be seen in detail below. The best-fit parameters are

$$
\begin{array}{cc}
\sigma_{u}=230 \mathrm{MeV}, & \sigma_{d}=170 \mathrm{MeV}, \\
\sigma_{g}=77 \mathrm{MeV}, & Q_{0}=0.75 \mathrm{GeV}, \\
\sigma_{H}=100 \mathrm{MeV}, & \alpha_{\mathrm{p} \pi}^{2}=0.45, \\
\alpha_{n \pi}^{2}=0.14, & \alpha_{\Lambda K}^{2}=0.05 .
\end{array}
$$

The normalization $\alpha_{B M}^{2}$ for the proton fluctuating to a baryon $B$ and a meson $M$ is defined as the fraction of the proton momentum taken by all partons $i$ in the fluctuation

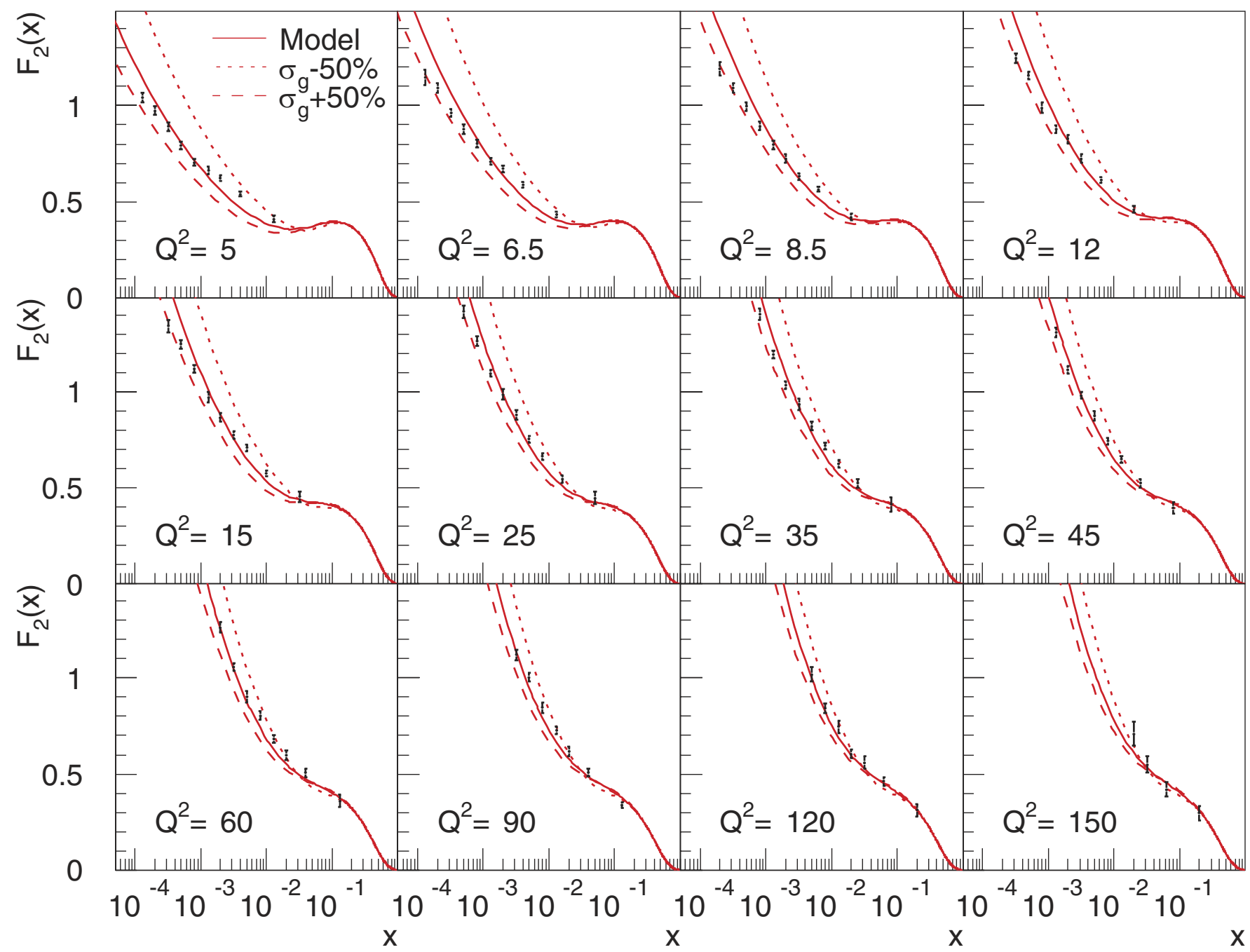

FIG. 5 (color online). The proton structure function $F_{2}\left(x, Q^{2}\right)$; H1 data [18] compared to our model with its sensitivity to a $\pm 50 \%$ variation of the width parameter $\sigma_{g}$ of the gluon distribution. 
$B M$,

$$
\alpha_{B M}^{2}=\int_{0}^{1} d x\left[\sum_{i \in B} x f_{i / B}(x)+\sum_{i \in M} x f_{i / M}(x)\right] .
$$

\section{A. Inclusive $\boldsymbol{F}_{\mathbf{2}}$ data}

The model was originally [5] used only to fit inclusive data, using a somewhat different definition for the hadronic fluctuations, and was shown to give a good fit. Now we are interested mainly in the model's description of asymmetries in the proton, and, hence, the $F_{2}$ data is used mainly to fix some model parameters which are not well restricted by asymmetry data, namely, the Gaussian width of the gluon distribution $\sigma_{g}$ and the starting scale $Q_{0}$. However, in view of the simplicity of the model, we still get a nice description of $F_{2}$ data from H1 [18], see Fig. 5. To show the sensitivity to the value of $\sigma_{g}$, we also show the result of varying this parameter with $\pm 50 \%$. The surprisingly low value for $\sigma_{g}$ obtained in the fit will be discussed in Sec. III E.

Since this data is restricted to relatively low $x$ values, we also compare to fixed-target NMC and BCDMS data $[16,17]$, which constrains the Gaussian widths of the $u$ and $d$ quark distributions, $\sigma_{u}$ and $\sigma_{d}$. This is shown in Fig. 6, which also shows the sensitivity of the fit to changing these parameters with $\pm 20 \%$. As seen in the figure, the $u$ width is quite well constrained by the large- $x$ data, while the $d$ width is less well constrained, and the data might account for a $\sigma_{d}$ which is up to $20 \%$ smaller. This is certainly true for the NMC data, which actually seem to prefer a smaller $d$ width. However, the solid curve describes the best-fit value due to the very small error bars on the BCDMS data, so we keep this as our main result in the following.

\section{B. The $W^{ \pm}$forward-backward asymmetry}

The forward-backward charged lepton asymmetry from $W^{ \pm}$decays in $p \bar{p}$ collisions at the Tevatron provides information on the large- $x$ distributions of $d$ and $u$ quarks. This is because the $W$ bosons are produced mainly through the processes $u \bar{d} \rightarrow W^{+}$and $d \bar{u} \rightarrow W^{-}$. If the $u$ distribution is harder than the $d$ distribution, we will get more $W^{+}$ than $W^{-}$in the direction of the proton beam and vice versa in the direction of the $\bar{p}$ beam, which is precisely what is observed. The data is for $Q^{2} \gtrsim M_{W}^{2}$ and $0.006<x<0.34$, corresponding via PQCD evolution to $0.01 \lesssim x \lesssim 0.6$ at the starting scale $Q_{0}^{2} \sim 1 \mathrm{GeV}^{2}$. We can therefore use the data on the charge asymmetry versus rapidity, expressed as

$$
A\left(y_{l}\right)=\frac{d \sigma^{+} / d y_{l}-d \sigma^{-} / d y_{l}}{d \sigma^{+} / d y_{l}+d \sigma^{-} / d y_{l}}
$$

to get further information on the Gaussian width of the $d$ quark distribution. The result, using our PQCD-evolved parton distributions in PYTHIA to simulate $W^{ \pm}$production via the processes $q \bar{q}^{\prime} \rightarrow W^{ \pm}, q \bar{q}^{\prime} \rightarrow g W^{ \pm}$, and $q g \rightarrow$ $W^{ \pm} q^{\prime}$, and subsequent decay $W^{ \pm} \rightarrow l^{ \pm} \nu_{l}$, is shown in Fig. 7. It is clear that the model reproduces the salient features of the data, in particular, when the $d$ width is reduced as preferred by the NMC data discussed above.

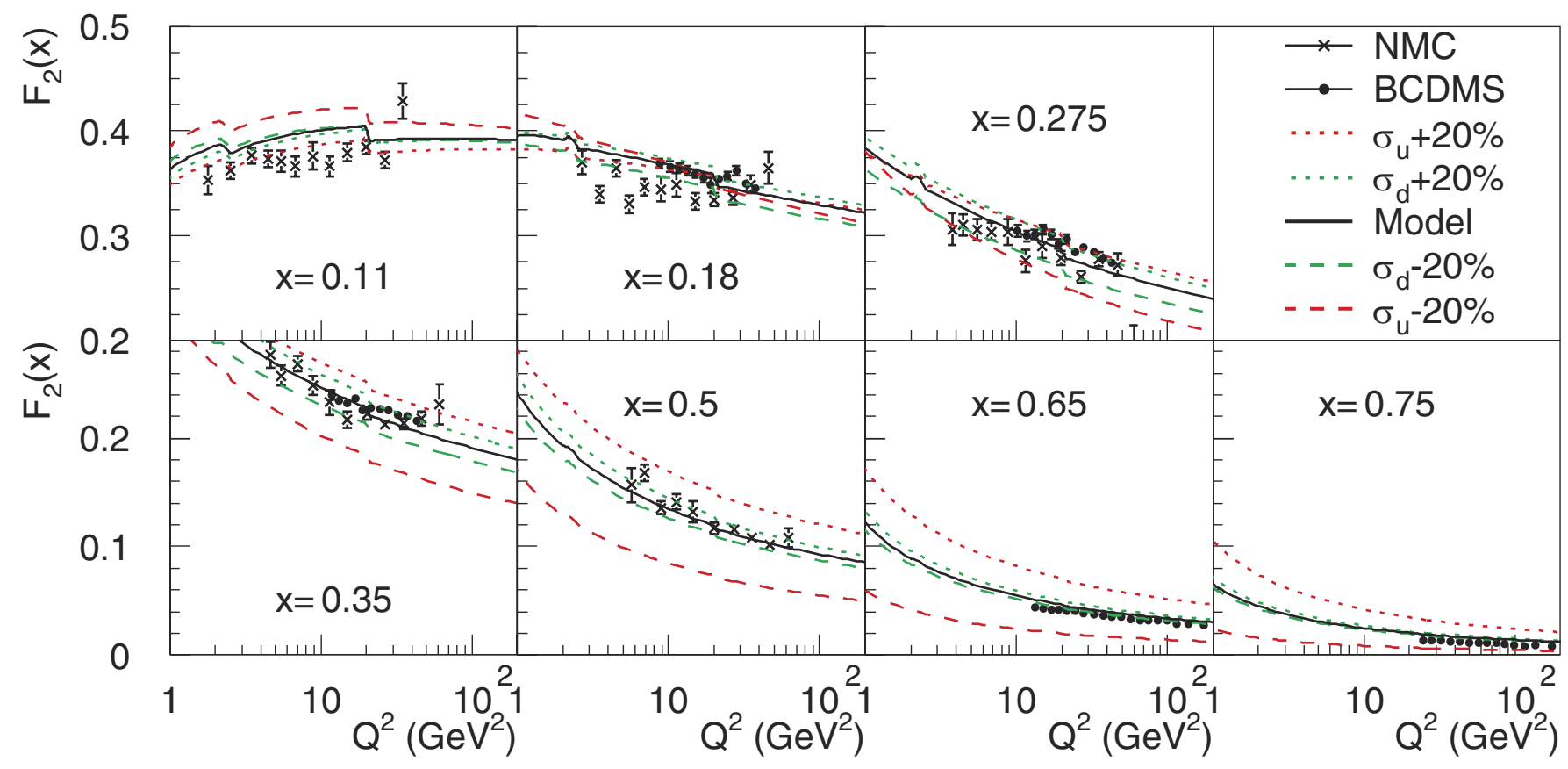

FIG. 6 (color online). The proton structure function $F_{2}\left(x, Q^{2}\right)$ for large $x$ values; NMC and BCDMS data [16,17] compared to our model, also showing the results of $\pm 20 \%$ variations of the width parameters $\sigma_{u}$ and $\sigma_{d}$ for the $u$ and $d$ valence distributions. 


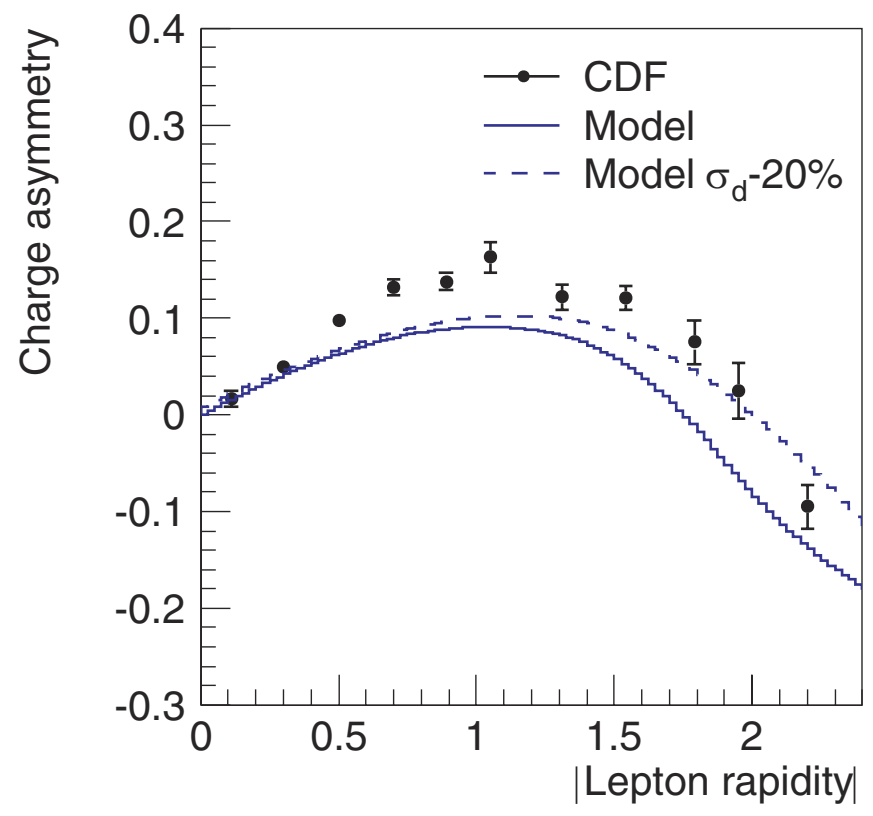

FIG. 7 (color online). The charge asymmetry versus rapidity, Eq. (13), for leptons from $W^{ \pm}$decays in $p \bar{p}$ collisions at the Tevatron [19] (with positive and negative lepton rapidity combined) compared to PYTHIA simulations using parton densities from our model, with best-fit parameters and a $20 \%$ reduced width of the valence $d$ quark distribution.

The difference between the widths of the $u$ and $d$ quarks $\left(\sigma_{u}=230 \mathrm{MeV}\right.$ and $\left.\sigma_{d}=135-170 \mathrm{MeV}\right)$ can perhaps be attributed to the Pauli blocking effect on the $u$ quarks as discussed above. Large differences in the intrinsic transverse momentum between the $u$ and $d$ quarks are indeed indicated by data from HERMES [22]. Also, the standard parametrizations of parton densities [3,4] result in harder spectra for the $u$ than for the $d$ valence distribution.

\section{The $\bar{d}-\bar{u}$ asymmetry}

Now we come to the important results of the model regarding the sea quark asymmetries. These result from the fluctuations of the proton to meson-baryon pairs. The first such asymmetry that we will investigate, and the one with most experimental data, is the difference between $\bar{u}$ and $\bar{d}$ distributions.

In this model, the $\bar{u}$ and $\bar{d}$ sea comes mainly from fluctuations of the proton into a nucleon and a pion, since these are the lowest energy fluctuations. This means that we will get an excess of $\bar{d}$ over $\bar{u}$, since $\left|p \pi^{0}\right\rangle$ is symmetric between $\bar{d}$ and $\bar{u}$, while $\left|n \pi^{+}\right\rangle$contains only $\bar{d}$ and no $\bar{u}$. If these were the only available fluctuations we would get from isospin Clebsch-Gordan coefficients, the ratio $\alpha_{p \pi}^{2} / \alpha_{n \pi}^{2}=C_{p \pi}^{2} / C_{n \pi}^{2}=1 / 2$. However, this is modified by the inclusion of $|\Delta \pi\rangle$ fluctuations (which have small mass suppression relative to the $|N \pi\rangle$ fluctuations). In the SU(6) scheme, the $\left|\Delta^{++} \pi^{-}\right\rangle$fluctuation has a larger Clebsch-Gordan coefficient than the $\left|\Delta^{0} \pi^{+}\right\rangle$, which increases the relative amount of $\bar{u}$ quarks. In our model, we take such higher-mass fluctuations into account by simply fitting the normalization parameters $\alpha_{p \pi}^{2}$ and $\alpha_{n \pi}^{2}$ using experimental data on the light sea.

The ratio $\bar{d} / \bar{u}$ can be extracted by comparing muon pair production through the Drell-Yan process, $q \bar{q} \rightarrow l^{+} l^{-}$, in $p p$ collisions with that in $p d$ collisions assuming isospin symmetry. Using parametrizations for the sum $\bar{d}(x)+$ $\bar{u}(x)$, the difference $\bar{d}(x)-\bar{u}(x)$ can also be extracted.

We have used data from the E866 Collaboration [20] to fit the parameters relevant for the $\bar{d}$ and $\bar{u}$ distributions. These parameters are the normalizations $\alpha_{p \pi}^{2}$ and $\alpha_{n \pi}^{2}$ and the Gaussian width $\sigma_{H}$. However, the sensitivity of the meson parton distributions to $\sigma_{H}$ is small, and, in particular, the position of the peak of the momentum distributions is quite independent of $\sigma_{H}$ and depends only on the mass of the fluctuation hadrons. For small $\sigma_{H} \lesssim 100 \mathrm{MeV}$, also the overall shape of the meson distributions is determined mainly by the hadron masses. For larger $\sigma_{H} \geq 100 \mathrm{MeV}$, the parton distributions inside the fluctuation hadrons are altered due to the kinematical limits in such a way as to keep the convoluted shape stable. Therefore, we take the value of $\sigma_{H}$ to be $100 \mathrm{MeV}$, which is preferred by the fit.

In the distribution of $\bar{d}(x)-\bar{u}(x)$ [or, equivalently $x \bar{d}(x)-x \bar{u}(x)$ ], the effect of symmetric $q \bar{q}$ pair production from gluons in the PQCD evolution is canceled, and, thus, the result depends only on the properties of the nonperturbative distributions at the starting scale, barring the uncertainties from the extraction of the data from experiments. Therefore, it is quite interesting to compare the experimental results for this distribution with the model results. As can be readily seen in Fig. 8(b), the pion distribution using the physical pion mass $m_{\pi}=140 \mathrm{MeV}$ (solid line) is too soft, peaking at $x \approx 0.05$, while the data peak around $x \simeq$ 0.1 . This cannot be cured using a larger $\sigma_{H}$, as explained above, but rather indicates that one should use a larger effective pion mass. Allowing this effective pion mass to be a free parameter in the fit, the best-fit result is that $m^{\text {eff }} \approx$ $400 \mathrm{MeV}$, as showed in Fig. 8 (dashed line). Note that the points driving the fit are the small- $x \bar{d} / \bar{u}$ data, which have the smallest errors.

One can think of several explanations for such a large effective pion mass. One could be that we need to include a relatively large fraction of heavier mesons, such as the $\rho$ mesons, in our description. This would be in accordance with the finding of a large $|N \rho\rangle$ component in the study [7] based on a meson cloud model. Another explanation would be that, since the pions have very nontypical hadron masses, the meson-baryon fluctuations involve more generic meson states, with the quantum numbers defined by the actual hadron states available, but with masses with some distribution which can be approximated with a meson mass of $400 \mathrm{MeV}$. In any case, the introduction of a larger pion mass without any other modifications of the model reduces the quality of the fit to the low- $x F_{2}$ data described in Sec. III A (the effect is comparable to changing the value 


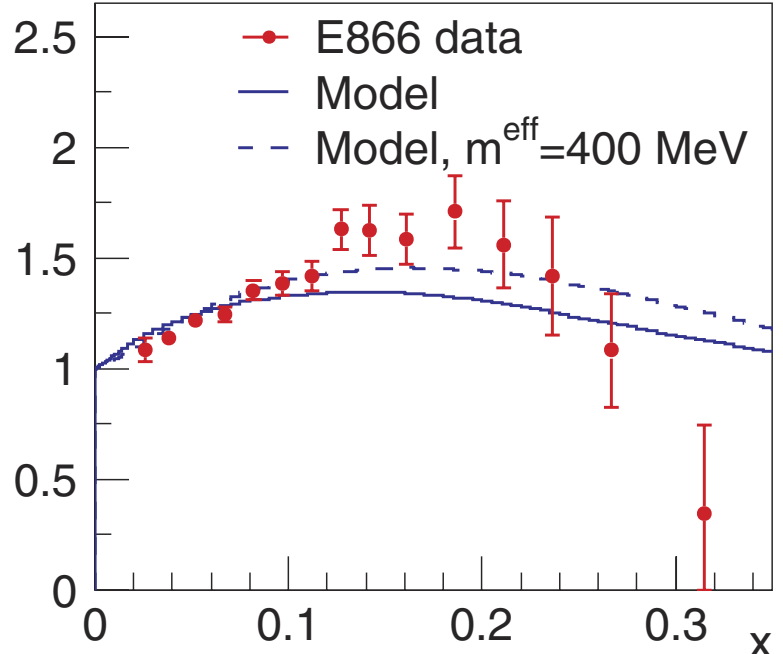

(a)

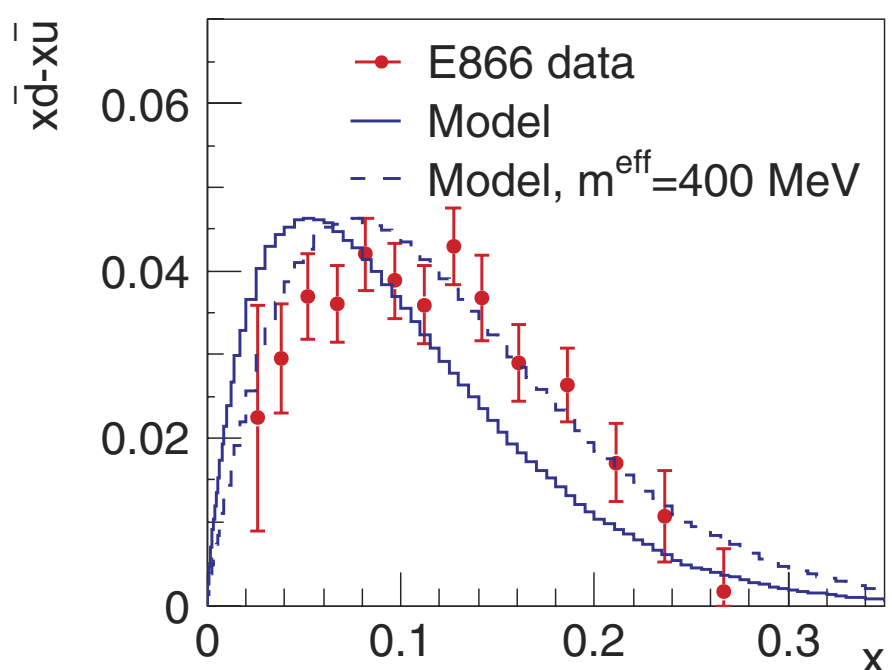

(b)

FIG. 8 (color online). Comparison between our model and data from the E866/NuSea Collaboration [20]: (a) $\bar{u}(x) / \bar{d}(x)$, (b) $d(x)-u(x)$. The solid line shows the results of the model using the physical pion mass, while the dashed line shows the results with an effective pions mass $m^{\text {eff }}=400 \mathrm{MeV}$ as discussed in the text. The parton distributions are evaluated at $Q^{2}=54 \mathrm{GeV}^{2}$.

of $\sigma_{g}$ by less than $+50 \%$ ), which points to the need of introducing more assumptions and parameters to get a perfect description of all data. Since our intention is not to get the best possible fit (as conventional parton density parametrizations), we refrain from doing this without a physics motivation related to our model.

\section{The strange sea asymmetry}

Another consequence of the model is an asymmetry between the strange and antistrange quark $x$ distributions in the nucleon, an aspect that we did a special study of in Ref. [13] and extend here. The reason for this asymmetry is that, in fluctuations with open strangeness, the $s$ quark is found in the baryon while the $\bar{s}$ quark is in the meson. Since the baryon, due to its higher mass, has a harder $x_{H}$ spectrum than the meson, we get a harder $x$ spectrum for the $s$ quark than the $\bar{s}$. This effect persists in spite of the fact that the $s$ distribution in the $\Lambda$ peaks at lower $x_{p}$ than the $\bar{s}$ distribution of the $K^{+}$; see [5] for examples of typical meson and baryon distributions from the model.

For simplicity, we assume that all fluctuations including strangeness (such as $\left|\Lambda K^{*}\right\rangle,|\Sigma K\rangle$ ) can be implicitly included in the $|\Lambda K\rangle$ fluctuation. Fluctuations where the $s \bar{s}$ pair is part of a meson wave function, thus giving a symmetric contribution, are neglected since they should be suppressed ( $\phi$ due to large mass and $\eta$ due to ClebschGordan coefficients). The resulting $s$ and $\bar{s}$ distributions are shown in Fig. 4(b).

Note that in this model the criterion that the nucleon should have zero total strangeness, $\int_{0}^{1}(s(x)-\bar{s}(x))=0$, is automatically fulfilled, since all strange fluctuations have the same number of $s$ quarks in the baryon as $\bar{s}$ quarks in the meson due to the sum rules ( 3 ) modified for the hadrons in question. This normalization is not changed when the parton distributions of the fluctuation are convoluted with the distributions of the fluctuation hadrons in the nucleon (except for the factor $\alpha_{B M}$, which is common to the baryon and the meson).

Since the normalization of the $|\Lambda K+\rangle$ fluctuation cannot be safely calculated, we take it as a free parameter which we fit to data from the CCFR Collaboration [21] on the averaged strange sea $(s+\bar{s}) / 2$, as shown in Fig. 9. Here perturbative QCD evolution to larger $Q^{2}$ shifts the original $s$ and $\bar{s}$ distributions to smaller $x$ and adds a symmetric sea arising from $g \rightarrow s \bar{s}$. The fit gives a $|\Lambda K+\rangle$ normalization $\alpha_{\Lambda K}^{2}=0.055$ such that $\int_{0}^{1} d x(x s(x)+x \bar{s}(x)) /$ $\int_{0}^{1} d x(x \bar{u}(x)+x \bar{d}(x)) \approx 0.5$; i.e. the strange sea momentum fraction at $Q_{0}^{2}$ is approximately half of that of a light sea quark, in agreement with the parton density analyzes in e.g. Ref. [23] and the meson cloud model study in Ref. [7].

This normalization of the nonperturbative strange quark sea means that the coefficient $\alpha_{\Lambda K}^{2}$ has essentially been scaled down with the fluctuation time $\Delta t \sim 1 / \Delta E$ relative to the light sea. This is a smaller suppression than $1 /(\Delta E)^{2}$ that one might have expected based on old-fashioned perturbation theory. On the other hand, this $s \bar{s}$ suppression is of similar magnitude as the ratio $P(s \bar{s}) / P(u \bar{u}) \approx 1 / 3$ of the probabilities for quark-antiquark production in phenomenological hadronization models, such as the Lund model [24]. Given that both cases concern nonperturbative $s \bar{s}$ pair production in a color field, this need not be surprising but indicates common features.

This $|\Lambda K+\rangle$ fluctuation results in an asymmetry $S^{-}=$ $\int_{0}^{1} d x(x s(x)-x \bar{s}(x))=0.00165 \quad$ at $\quad Q^{2}=20 \mathrm{GeV}^{2}$. However, this result is not altogether independent of the details of the model. The precise definition for the upper limit of the struck quark virtuality discussed in Sec. II C 1 , 


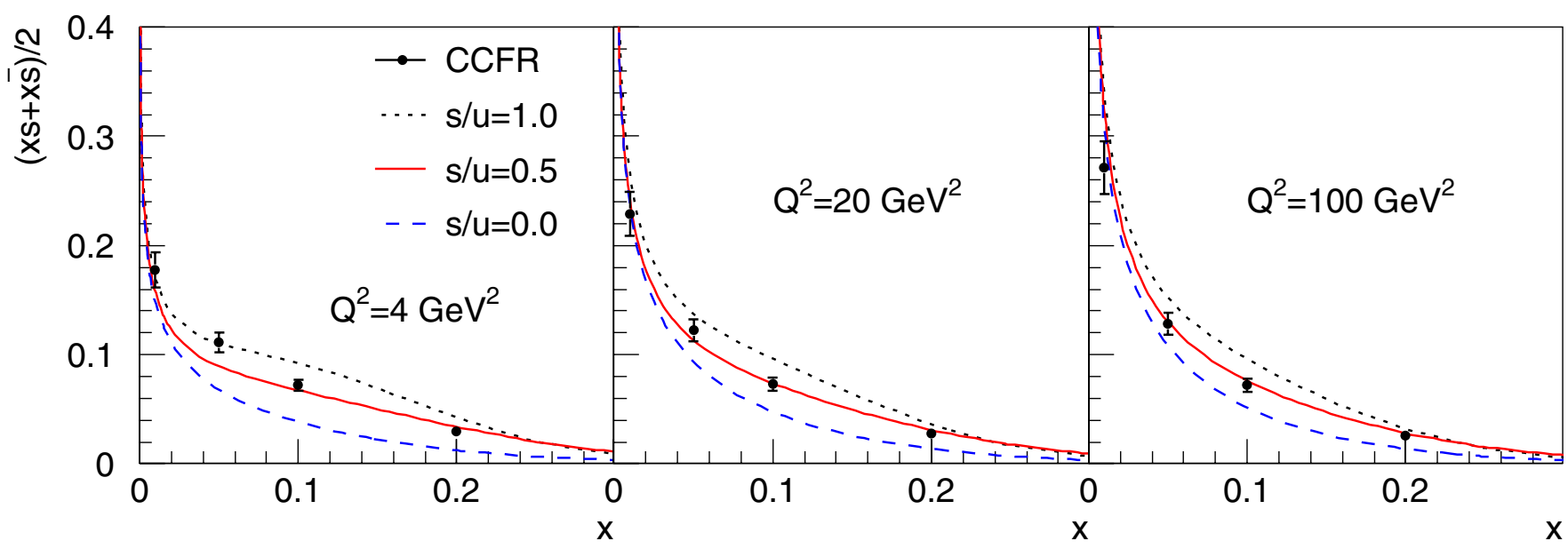

FIG. 9 (color online). CCFR deep inelastic scattering data [21] on the strange sea distribution $(x s(x)+x \bar{s}(x)) / 2$ in the nucleon at different $Q^{2}$ compared to our model based on $|\Lambda K\rangle$ fluctuations [with normalization $s / u=(s+\bar{s}) /(\bar{u}+\bar{d})$ as discussed in the text] at a low scale and evolved to larger $Q^{2}$ with perturbative QCD that also adds a symmetric perturbative $s \bar{s}$ component.

item 2 , and the width of the $d$ distribution (which gives the width of the valence quark distributions in $\Lambda$ and $K$ ) both have influence on the value. Using both definitions for the limit and allowing the $d$ width to vary between the values used in Fig. 7, we get a range of values for the asymmetry

$$
0.0010 \leq S^{-} \leq 0.0023
$$

An asymmetry in the momentum distributions of $s$ and $\bar{s}$ is especially interesting in view of the NuTeV anomaly. In the NuTeV experiment [14], the value of $\sin ^{2} \theta_{W}$ was extracted from neutral and charged current cross sections of neutrinos and antineutrinos. The value they find differs by about $3 \sigma$ from the value obtained in standard model fits to data from other experiments: $\sin ^{2} \theta_{W}^{\mathrm{NuTeV}}=0.2277 \pm$ 0.0016 , while $\sin ^{2} \theta_{W}^{\mathrm{SM}}=0.2227 \pm 0.0004$. A number of possible explanations [25] for this discrepancy have been suggested, both in terms of extensions to the standard model and in terms of effects within the standard model. One explanation within the standard model is that, since the neutrinos and antineutrinos interact differently with $s$ and $\bar{s}$ quarks, an asymmetry $S^{-} \neq 0$ shifts the NuTeV result. In order to facilitate the calculation of such a shift, NuTeV has published a folding function $F(x)$ [26] to account for their analysis and give the shift in the extracted value of $\sin ^{2} \theta_{W}$, where the parton densities are to be calculated at $Q^{2}=20 \mathrm{GeV}^{2}$. Using this, we get the shift $\Delta \sin ^{2} \theta_{W}=\int_{0}^{1} d x s^{-}(x) F(x)=-0.0017$, corresponding to a reduction of the significance of the anomaly with 1 standard deviation. Our results for $s^{-}(x)=x s(x)-$ $x \bar{s}(x)$ and $s^{-}(x) F(x)$ are shown in Fig. 10. The range of values of $S^{-}$of Eq. (14) corresponds to $-0.0024 \leq$ $\Delta \sin ^{2} \theta_{W} \leq-0.00097$ for the shift in $\sin ^{2} \theta_{W}^{\mathrm{NuTeV}}$. This reduces the anomaly to between $1.6 \sigma$ and $2.4 \sigma$ away from the standard model value.

This important topic has been investigated previously. On the theoretical side we note that it has recently been shown [27] that higher order perturbative effects give a negative contribution to $S^{-}$, although significantly smaller than our positive nonperturbative effect. Within the meson cloud model of Ref. [28], based on exponentially suppressed $\Lambda K$ fluctuations, an inconclusive result on the sign of $S^{-}$was obtained and a strange sea that was too small to affect the NuTeV anomaly. The meson cloud model in Ref. [7] obtained that the $s$ quark has the softer spectrum than the $\bar{s}$, corresponding to a negative $S^{-}$. Using a light-cone two-body wave function model applied to $\Lambda K$ fluctuations [29], a positive result similar to ours was obtained in Ref. [30], and in Ref. [31] an even larger reduction of the anomaly than ours was found using an effective chiral quark model. Unfortunately, none of these studies provides comparisons with the measured strange sea, making the significance of their results difficult to assess.

The experimental situation is at present unclear. In Ref. [32], a positive $S^{-}$was favored based on several earlier experiments, whereas NuTeV [26,33] obtains the opposite sign based on their own data. However, the global

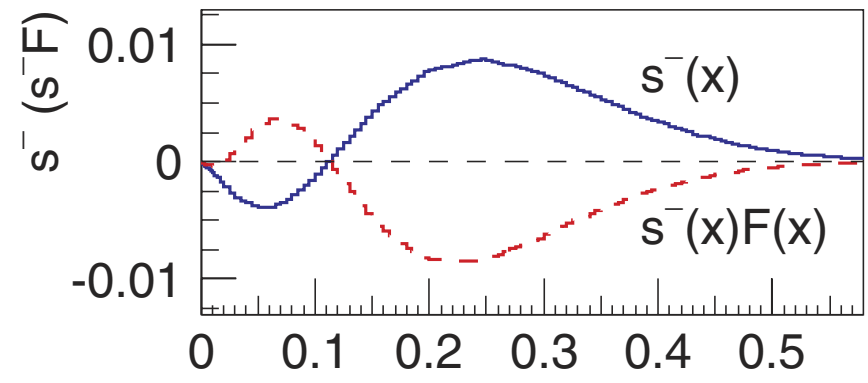

$\mathrm{X}$

FIG. 10 (color online). The strange sea asymmetry $s^{-}(x)=$ $x s(x)-x \bar{s}(x)\left(\right.$ at $Q^{2}=20 \mathrm{GeV}^{2}$ ) from the model and combined with the function $F(x)$ accounting for NuTeV's analysis giving $\Delta \sin ^{2} \theta_{W}=\int_{0}^{1} d x s^{-}(x) F(x)=-0.0017$. 
analysis in Ref. [34] of the $s-\bar{s}$ asymmetry, including the $\mathrm{NuTeV}$ data as well as the CCFR data and using a very general functional form for $s^{-}(x)$, gives a best-fit value for the asymmetry $S^{-}$of the same magnitude and sign as ours.

It is clear that an asymmetry in the strange quark sea of the nucleon arises naturally in models where nonperturbative sea quark distributions originate from hadronic fluctuations of the nucleon. Our model, which reproduces the measured strange sea, reduces the NuTeV anomaly to a level which does not give a significant indication of physics beyond the standard model.

\section{E. Comparison to standard parametrizations}

Let us first note that it is a nontrivial result that the many different data sets shown above can be well described by a model with only the eight parameters in Eq. (11). The model provides all the different parton distributions in $x$ at $Q_{0}^{2}$, i.e. $u_{v+\text { sea }}, d_{v+\text { sea }}, g, \bar{u}, \bar{d}, s, \bar{s}$. This is achieved with only four shape parameters (the $\sigma$ 's) and three normalization parameters (the $\alpha_{M B}$ 's), i.e. significantly fewer shape and normalization parameters than distributions.

The simplest possible conventional parametrization based on counting rules [9], $x f_{i}(x)=A_{i} x(1-x)^{a_{i}}$, cannot be as economic in the number of parameters and still allow different shapes of different sea distributions. Considering only valence and light sea distributions and neglecting the strange sea, we were able to get reasonable fits of the same data sets with eight free parameters [when applying the constraints in Eqs. (3) and (4)]. However, the fitted values for the exponents $a_{i}$ for the sea quarks and the gluon turn out more than twice as large as expected from the counting rules (using the expected values gives a poor description of the data). With our model we obtain a generally better description of the data which is achieved with fewer parameters. Moreover, a physically motivated model whose parameters have a physical interpretation can give new insights about the unknown nonperturbative QCD dynamics embodied in the parton distributions.

The standard parametrizations of parton densities, such as CTEQ [3] and MRST [4], have many more parameters (typically 20 for the $x$ shapes and additional ones for normalizations) and provide high-quality fits to large sets of different kinds of data. It is, therefore, interesting to compare the parton densities resulting from our model with such standard parametrizations.

In Fig. 11 we show our parton densities together with the uncertainty band of the CTEQ6M distributions [3] at the low scale $Q^{2}=1.3 \mathrm{GeV}^{2}$. Several features of the plot are striking:

(1) For large and intermediate $x$, our valence quark as well as sea quark distributions from the model agree quite well with those from CTEQ6M.

(2) Our gluon distribution is slightly lower at large $x$ and much larger at small $x$.

(3) For small $x \lesssim 10^{-2}$ the quark and antiquark distributions of CTEQ are clearly higher and growing faster as $x \rightarrow 0$.

The last two points are connected; if there is a deficit in low- $x$ sea quarks of nonperturbative origin in our model, this must be compensated by perturbatively generated sea

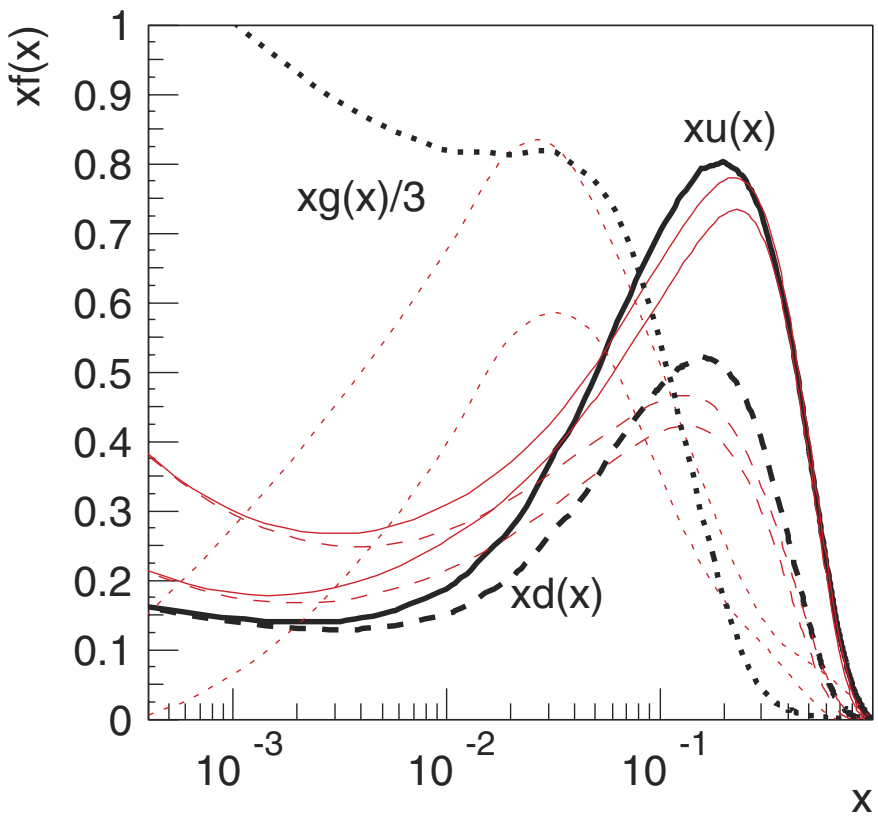

(a)

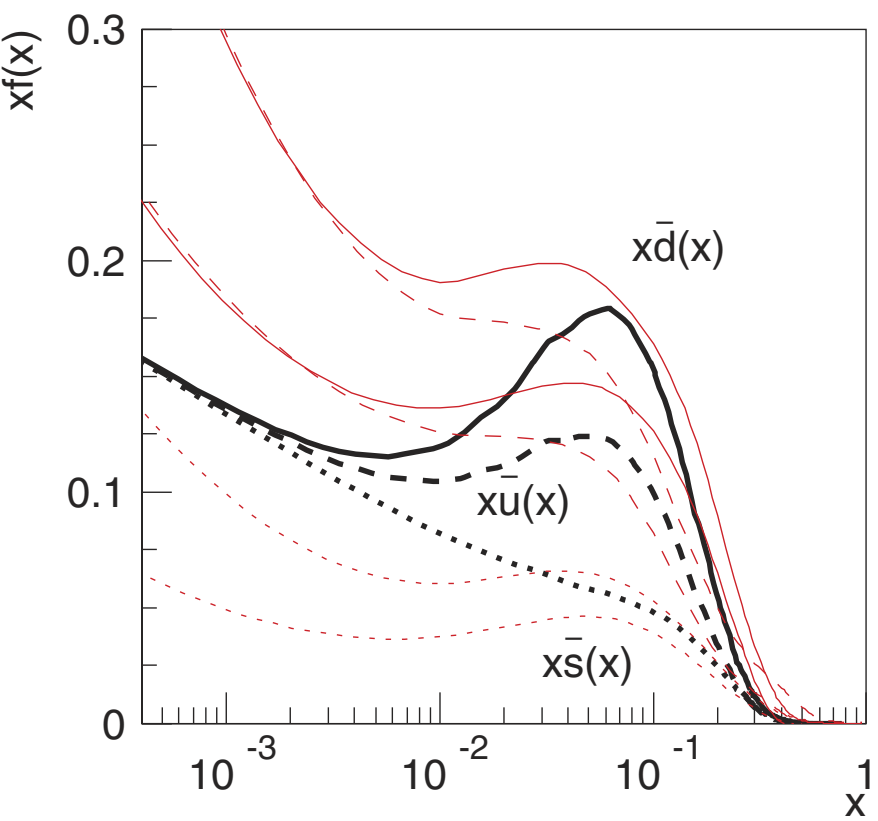

(b)

FIG. 11 (color online). Comparison between parton densities at $Q^{2}=1.3 \mathrm{GeV}^{2}$ from our model (thick lines) and the CTEQ6M uncertainty band [3] (plotted using the Durham HEPDATA server [35]). Note the different scales on the axes of (a) and (b) and that the gluon distributions are divided by 3 to fit in the plot. 
quarks from $g \rightarrow q \bar{q}$ in the PQCD evolution in order to fit HERA $F_{2}$ data. This requires a large gluon density at small $x$ and a low starting scale $Q_{0}^{2}$, which combined with the momentum sum rule equation (4) gives the low width $\sigma_{g}$ of the gluon distribution. If instead of using next-to-leading order QCD evolution equations, we use only leading order ones, then the evolution is slower, which makes the fit to data prefer an even lower starting scale $Q_{0}^{2}$ and a still more peaked gluon distribution at small $x$, i.e. a smaller $\sigma_{g}$, in order to develop a large enough $q \bar{q}$ sea at low $x$. Thus, these two model parameters depend on the order used in the perturbative evolution, whereas the other parameters are not affected much. Furthermore, a worse fit to data is obtained with the leading order evolution.

The Gaussian momentum fluctuations in our model leads to parton number densities that go to a constant as $x \rightarrow 0$ and, hence, momentum densities going to zero as $x \rightarrow 0$. The increase of the momentum distributions at $x \rightarrow$ 0 seen in Fig. 11 is thus caused by PQCD evolution from the starting scale $Q_{0}^{2}$, which is forced to be very low. We cannot, however, get a sea quark distribution rising as fast as the $x^{-0.3}$ shape obtained by CTEQ [as seen in Fig. 11(b)] at their $Q_{0}=1.3 \mathrm{GeV}$, without an accompanying rise in the $x g(x)$ density. This indicates that, besides mesonbaryon fluctuations, there is some additional source of $q \bar{q}$ pairs. One possibility, which seems quite natural, would be a nonperturbative "gluon splitting" process similar to the one in PQCD evolution. However, the precise form and effect of such a process is not known. Such a contribution would not have any large effect on the asymmetries studied in this work, since the asymmetry data are found at larger $x$ and $Q^{2}$. Therefore, we have chosen not to complicate our model by further assumptions and parameters to add such a conceivable component.

\section{CONCLUSIONS}

We have presented a model based on Gaussian momentum fluctuations and hadronic fluctuations of baryons into baryon-meson pairs. The resulting parton momentum distributions describe a wealth of data surprisingly well in view of the simplicity of the model. In this study, we have focused on different asymmetries of quark distributions in the proton; most notably, the $u-d$ asymmetry obtained from the $W^{ \pm}$forward-backward asymmetry at the Tevatron and the $\bar{d}-\bar{u}$ asymmetry measured in $p p$ and $p d$ scattering. From the phenomenological success of the model, we can draw some overall conclusions:

(i) The Gaussian, based on the law of large numbers, used for the momentum distribution may justify a statistical description of the nonperturbative forces confining partons in hadrons. (ii) Asymmetries in the quark sea can be explained by hadronic fluctuations, such as $|p\rangle=\alpha_{0}\left|p_{0}\right\rangle+$ $\alpha_{p \pi}\left|p \pi^{0}\right\rangle+\alpha_{n \pi}\left|n \pi^{+}\right\rangle+\ldots+\alpha_{\Lambda K}\left|\Lambda K^{+}\right\rangle+\ldots$

From the details of our study, we can make some further assertions:

(i) The difference in shape between the $u$ and $d$ valence distributions of the proton corresponds to a difference in the widths of their momentum distributions, which may be interpreted as a difference in the spacial region available due to Pauli blocking.

(ii) Comparison with the experimental result for the distribution of $\bar{d}(x)-\bar{u}(x)$ indicates that a larger effective mass is needed for the pion in the $|N \pi\rangle$ states, which may be interpreted as an effective way of also including fluctuations with heavier mesons.

(iii) Besides fluctuations into hadronic states, some other mechanism seems to be needed to explain the rise in the sea $q \bar{q}$ distributions as $x \rightarrow 0$, used in standard parametrizations of parton distributions.

(iv) An asymmetry between the strange and antistrange nucleon sea is unavoidable, although the magnitude of the asymmetry $S^{-}=\int_{0}^{1} d x(x s(x)-x \bar{s}(x))$ cannot be stated more precisely than $0.0010 \leq S^{-} \leq$ 0.0023 . This reduces the NuTeV anomaly from 3 to about 2 standard deviations, leaving no significant indication for physics beyond the standard model.

Although our model invoke similar basic hadronic fluctuations as in meson cloud models, it differs in important aspects, primarily concerning the basis for the momentum distributions of both the hadrons in the fluctuations and the partons in the hadrons. As discussed above, our model is also more economical in terms of using fewer parameters than even the simplest parametrizations of parton densities. Our model provides the parton distributions for all light quarks, as well as the strange sea and gluons, with altogether only four parameters giving the $x$ shapes and three parameters for normalizations.

Finally, we note that this model could be used to develop a library of parton density functions for more general usage. So far, we have not done this since our intention has not been high precision functions to be folded with parton level cross sections in practical calculations. Instead, our primary aim has been to gain understanding of the nonperturbative QCD dynamics of the bound state nucleon that is embodied in the parton density functions at the low scale $Q_{0}^{2}$, which are otherwise just parametrized.

\section{ACKNOWLEDGMENTS}

This research was supported by the Swedish Research Council. 
[1] J.C. Collins and D.E. Soper, Nucl. Phys. B194, 445 (1982); J. C. Collins, D. E. Soper, and G. Sterman, Nucl. Phys. B261, 104 (1985); B308, 833 (1988); Phys. Lett. B 438, 184 (1998); G. T. Bodwin, Phys. Rev. D 31, 2616 (1985); 34, 3932(E) (1986).

[2] V. N. Gribov and L. N. Lipatov, Sov. J. Nucl. Phys. 15, 438 (1972); G. Altarelli and G. Parisi, Nucl. Phys. B126, 298 (1977); Yu.L. Dokshitzer, Sov. Phys. JETP 46, 641 (1977).

[3] J. Pumplin, D. R. Stump, J. Huston, H. L. Lai, P. Nadolsky, and W. K. Tung, J. High Energy Phys. 07 (2002) 012.

[4] A. D. Martin, R. G. Roberts, W. J. Stirling, and R. S. Thorne, Phys. Lett. B 531, 216 (2002).

[5] A. Edin and G. Ingelman, Phys. Lett. B 432, 402 (1998); Nucl. Phys. B Proc. Suppl. 79, 189 (1999).

[6] S. Kumano, Phys. Rep. 303, 183 (1998); R. Vogt, Prog. Part. Nucl. Phys. 45, S105 (2000).

[7] H. Holtmann, A. Szczurek, and J. Speth, Nucl. Phys. A596, 631 (1996).

[8] G. Ingelman et al., Nucl. Phys. B206, 239 (1982); J. J. Aubert et al. (European Muon Collaboration), Phys. Lett. 119B, 233 (1982); M. Arneodo et al. (European Muon Collaboration), Phys. Lett. 149B, 415 (1984).

[9] S. J. Brodsky and G. R. Farrar, Phys. Rev. Lett. 31, 1153 (1973).

[10] For a review, see T. H. Bauer et al., Rev. Mod. Phys. 50, 261 (1978); 51, 407(E) (1979).

[11] J. J. Sakurai and D. Schildknecht, Phys. Lett. 40B, 121 (1972).

[12] J. Alwall and G. Ingelman, Phys. Lett. B 596, 77 (2004).

[13] J. Alwall and G. Ingelman, Phys. Rev. D 70, 111505(R) (2004).

[14] M. Goncharov et al. (NuTeV Collaboration), Phys. Rev. D 64, 112006 (2001); G.P. Zeller et al. (NuTeV Collaboration), Phys. Rev. Lett. 88, 091802 (2002); 90,
239902(E) (2003).

[15] M. A. J. Botje, Zeus Note 97-006, 1997. See also http:// www.nikhef.nl/ h24/qcdnum/.

[16] M. Arneodo et al. (New Muon Collaboration), Phys. Lett. B 364, 107 (1995).

[17] A.C. Benvenuti et al. (BCDMS Collaboration), Phys. Lett. B 223, 485 (1989).

[18] C. Adloff et al. (H1 Collaboration), Eur. Phys. J. C 21, 33 (2001).

[19] F. Abe et al. (CDF Collaboration), Phys. Rev. Lett. 81, 5754 (1998).

[20] R.S. Towell et al. (FNAL E866/NuSea Collaboration), Phys. Rev. D 64, 052002 (2001).

[21] A. O. Bazarko et al. (CCFR Collaboration), Z. Phys. C 65, 189 (1995).

[22] A. Jgoun et al. (HERMES Collaboration), hep-ex/ 0107003.

[23] A. D. Martin et al., Eur. Phys. J. C 4, 463 (1998).

[24] B. Andersson et al., Phys. Rep. 97, 31 (1983).

[25] S. Davidson et al., J. High Energy Phys. 02 (2002) 037.

[26] G. P. Zeller et al. (NuTeV Collaboration), Phys. Rev. D 65, 111103 (2002); 67, 119902(E) (2003).

[27] S. Catani, D. de Florian, G. Rodrigo, and W. Vogelsang, Phys. Rev. Lett. 93, 152003 (2004).

[28] F. G. Cao and A. I. Signal, Phys. Lett. B 559, 229 (2003).

[29] S. J. Brodsky and B. Q. Ma, Phys. Lett. B 381, 317 (1996).

[30] Y. Ding and B. Q. Ma, Phys. Lett. B 590, 216 (2004).

[31] Y. Ding, R. G. Xu, and B. Q. Ma, Phys. Lett. B 607, 101 (2005).

[32] V. Barone et al., Eur. Phys. J. C 12, 243 (2000).

[33] D. Mason et al. (NuTeV Collaboration), hep-ex/0405037.

[34] F. Olness et al., Eur. Phys. J. C 40, 145 (2005).

[35] The Durham HEPDATA PDF server is found at http:// durpdg.dur.ac.uk/hepdata/pdf.html. 